\title{
Model-based Calibration of a Range Camera*
}

\author{
J.-A. Beraldin, M. Rioux, F. Blais and G. Godin \\ Institute for Information Technology \\ National Research Council Canada \\ Ottawa, Ontario, Canada K1A 0R6
}

\author{
R. Baribeau \\ Canadian Conservation Institute \\ Department of Communications \\ Ottawa, Ontario, Canada K1A 0C8
}

\begin{abstract}
This paper introduces a new method for the calibration of a range camera based on active triangulation. The technique is based on a model derived from the geometry of the synchronized scanner. From known positions of a calibration bar, a logistic equation is fitted with values of spot positions read from a linear position detector at a number of angular positions of a scanning mirror. Furthermore, with an approximate form of the general equations describing the geometry, a series of design guidelines are derived to help a designer conduct a preliminary study of a particular range camera. Experimental results demonstrating the technique are found to compare favorably with theoretical predictions.
\end{abstract}

\section{Introduction}

Among the many techniques proposed to extract three-dimensional information from a scene, active triangulation is used in applications such as automatic welding, measurement and reproduction of objects, and inspection of printed circuit boards [1]-[2]. An innovative approach, based on triangulation using a synchronized scanning scheme, was introduced by Rioux [3] to allow very large fields of view with small triangulation angles without compromising on resolution. With smaller triangulation angles, a reduction of shadow effects is inherently achieved. Implementation of this triangulation technique by an autosynchronized scanner approach gives a considerable reduction in the optical head size compared to standard triangulation methods. A 3-D profile of a surface is captured by scanning a laser beam onto a scene by way of an oscillating mirror, collecting the light that is scattered by the scene in synchronism with the projection mirror, and, finally, focusing this light onto a linear position detector. Figure 1 depicts the synchronization effect produced by the double-sided mirror. This measurement process yields two quantities per sampling interval: one is for the angular position of the mirror and one for the position of the laser spot on the position detector. Owing to the shape of the coordinate system spanned by these variables (see Fig. 2a), the resultant images are not compatible with the coordinate systems used by most geometric image processing algorithms. A re-mapping of these variables to

\footnotetext{
0 *NRC 33205-11th IAPR Int. Conf. on Pat. Rec.(1992), 163-
} 167

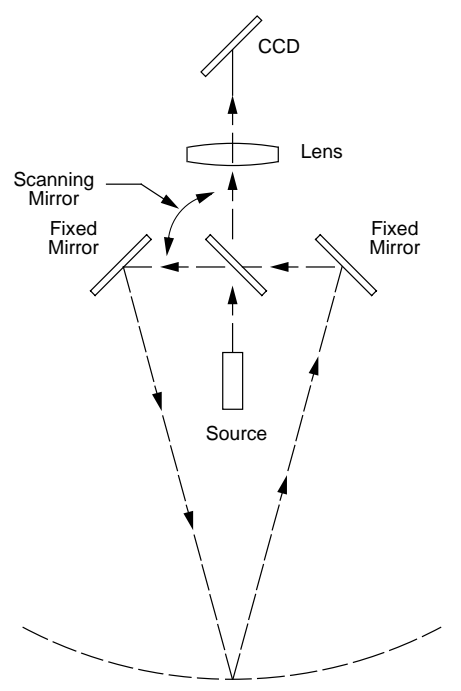

Figure 1: Auto-synchronized scanning geometry.

a more common coordinate system like a rectangular system is therefore required.

In [4], Bumbaca et al. present a method to calibrate a range finder without assuming any re-mapping function for the geometric distortions. The authors use a calibration bar composed of two sections: one having a uniform reflectance and one made of equidistant fiducial markings of lower reflectance, painted onto the surface. By constructing two tables from known positions of this calibration bar in space, they correct range and longitudinal distortions. The calibration bar is moved so as to define a rectangular coordinate system as depicted in Fig. 2b. Without knowledge of the distortion laws, any attempt to reduce the random noise present in the raw data to reveal the remapping function inherent in the range finder geometry becomes an art. Archibald et al. [5] propose replacing one of the tables by a series of linear equations fitted from data taken along the scan angles (Fig. 2c).

It is the goal of this article to extend the method presented in [4]-[5] and to introduce a novel method and procedure for the calibration of a range camera based upon the synchronization principle. Section 2 gives a description of the optical arrangement and presents the equations that describe the geome- 


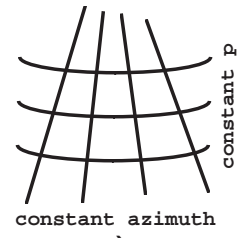

a)

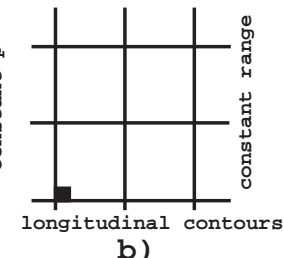

b)

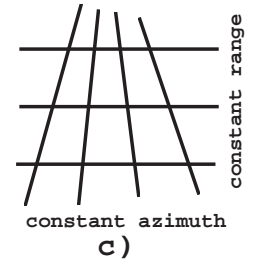

C)
Figure 2: Affine representations: (a) constant $\mathrm{p}$ and azimuth contours, (b) rectangular, (c) constant range and azimuth.

try from which a model is defined. These equations are used as design tools to characterize the volume of measurement and the spatial resolution, and to give some guidelines for a precise calibration of a range camera. Some experimental results obtained from a range finder intended for space applications are presented. Finally, a discussion of several advantages and limitations of the analysis and the proposed technique follows.

\section{Range camera equations}

Figure 3 shows the geometry used for the triangulation where the projection and collection axes have been unfolded. The dotted lines depict the static geometry, i.e., for $(\theta=0)$. Here, the scanning angle $\theta$ is measured from these dotted lines. Most triangulationbased range finders take advantage of the Scheimpflug optical arrangement [6]. The equations relating the spot position $p$ to the location of a point on the projection axis can be found from Fig. 3 in the following manner. Here, the scanning mirror has been temporarily removed and a pinhole model for the lens has been assumed. A rectangular coordinate system ha been located on the axis joining the equivalent position of the respective pivot of the projection and collection axes. These two positions are represented by large circles on the $X$ axis. The $Z$ axis extends from a point midway between them towards infinity. Superimposed on the figure is the equivalent geometry for a synchronized rotation of the projection and collection axes by an angle $\theta$.

The synchronized geometry implies that, for a spot position $p=0$ (point $\mathbf{A}$ on Fig. 3), the acute angle between the projection and collection paths is equal to a constant $\gamma$. From this, all the other angles can be inferred. Two sets of similar triangles OAC-OED and, OBC-OFD can be identified. From these, the following relation is extracted,

$$
\frac{R_{p}(\theta)-R_{-\infty}(\theta)}{R_{0}(\theta)-R_{-\infty}(\theta)}=\frac{P_{\infty}}{P_{\infty}-p}
$$

where $p$ is the spot position on the detector (detection axis), e.g., CCD, and for a given scanner angle $\theta$, $R_{p}(\theta)$ is the distance $R(\theta)$ along the projection axis corresponding to $p, R_{-\infty}(\theta)$ is the location of the vanishing point on the projection axis, and $R_{0}(\theta)$ is the location corresponding to $p=0 . P_{\infty}$ is the location on the position detector of the vanishing point on the

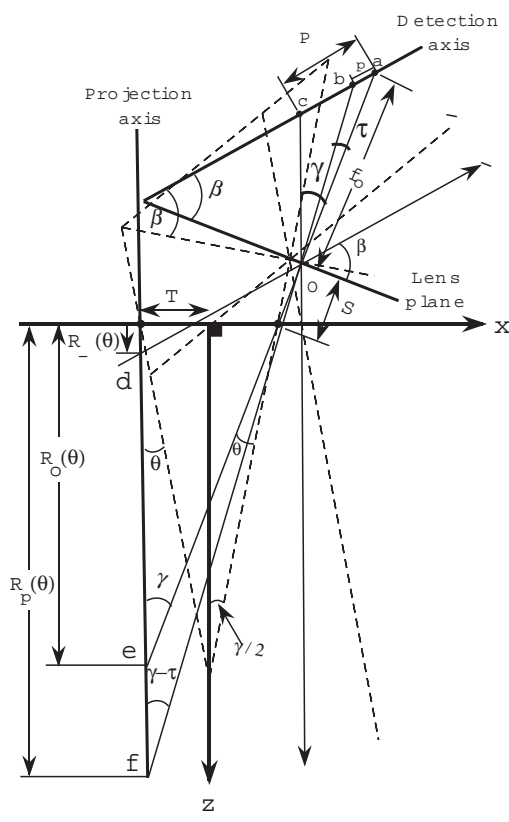

Figure 3: Un-folded geometry.

detection axis:

$$
P_{\infty}=f_{0} \frac{\sin (\gamma)}{\cos (\beta-\gamma)}=\frac{\left(f_{0}-f\right)}{\sin (\beta)}
$$

where $f$ is the focal length of the lens, $f_{0}$ is the effective distance of the position detector to the imaging lens, $\beta$ is the tilt angle of the position detector, and $\gamma$ is the triangulation angle.

The transformation of eq. (1) to an $(X, Z)$ representation is computed from the fact that two points $\mathbf{D}$ and $\mathbf{E}$ and a third point $\mathbf{F}$ belong to the same straight line if the vectors $\overrightarrow{\mathbf{D E}}$ and $\overrightarrow{\mathbf{D F}}$ are linearly dependent. Hence,

$$
\frac{X_{p}(\theta)-X_{-\infty}(\theta)}{X_{0}(\theta)-X_{-\infty}(\theta)}=\frac{Z_{p}(\theta)-Z_{-\infty}(\theta)}{Z_{0}(\theta)-Z_{-\infty}(\theta)}=\frac{P_{\infty}}{P_{\infty}-p}
$$

The above equations are decomposable in both orthogonal directions, i.e.,

$$
\begin{aligned}
& x(p, \theta)=X_{-\infty}(\theta)+P_{\infty} \frac{X_{0}(\theta)-X_{-\infty}(\theta)}{P_{\infty}-p} \\
& z(p, \theta)=Z_{-\infty}(\theta)+P_{\infty} \frac{Z_{0}(\theta)-Z_{-\infty}(\theta)}{P_{\infty}-p}
\end{aligned}
$$

These linear fractional equations, also known as logistic equations, emphasize the nature of the Scheimpflug geometry, that is, the limiting response $\left(R=R_{-\infty}\right)$ for $p$ as it approaches $-\infty$ (collection path parallel to the position detector) and $\left(p=P_{\infty}\right)$ for $R$ as it approaches $+\infty$ (projected ray parallel to collection path). 
The coordinates of points D, E, F are

$$
\begin{aligned}
X_{-\infty}(\theta)= & -\frac{T \cos (\beta-2 \theta)+S \cos (\beta) \sin (\gamma / 2-\theta)}{\cos (\beta-\gamma)} \\
Z_{-\infty}(\theta)= & \frac{T(\sin (\beta-2 \theta)+\sin (\beta-\gamma))}{\cos (\beta-\gamma)} \\
& -\frac{S \cos (\beta) \cos (\gamma / 2-\theta)}{\cos (\beta-\gamma)} \\
X_{0}(\theta)= & -T \frac{\sin (2 \theta)}{\sin (\gamma)} \\
Z_{0}(\theta)= & T \frac{\cos (2 \theta)+\cos (\gamma)}{\sin (\gamma)}
\end{aligned}
$$

where $S$ is the distance between the lens and the effective position of the collection axis pivot and $T$ is half the distance between the projection and collection pivots. These equations constitute the basis for the derivation of the design tools and calibration method presented in Section 3 .

\section{Proposed calibration method}

Once the equations describing the geometry are known, one can estimate some of the most important characteristics of a particular design.

\subsection{Volume of view}

Usually one begins with a given field of view and resolution for a particular application and uses the static geometry $(\theta=0)$ to evaluate those numbers. At this point, laser beam propagation must also be considered. Then, eqs. (4)-(9) are computed for a particular scan width and position detector size. This process is repeated until the system specifications have been met.

\subsection{Spatial resolution}

Two methods can be used to estimate the resolution. The first, given below, is the result of the application to $(\mathbf{x}, \mathbf{z})$ of the law of propagation of errors due to the measurement of $(\mathbf{p}, \boldsymbol{\Theta})$ through eqs. (4) and (5). The second method is the actual calculation of the joint density function of $\mathbf{x}=g(\mathbf{p}, \Theta)$ and $\mathbf{z}=h(\mathbf{p}, \Theta)$. This method allows for a full characterization of the two random variables $\mathbf{x}$ and $\mathbf{z}$ taken jointly. This result will be reported separately.

Blais [7] has designed a galvanometer controller that achieves a peak-to-peak error of 1 part in 5000 on a uni-directional scan for an optical angle of $30^{\circ}$, i.e., $\sigma_{\theta} \simeq 0.0001^{\circ}$. The measurement of $p$ is in practice limited by the laser speckle impinging on the CCD position detector. Baribeau and Rioux [8] predicted that such noise behaves like a Gaussian process and the estimated rms fluctuation of $p$ determined by the noise is approximately

$$
\sigma_{\mathrm{p}}=\frac{1}{\sqrt{2 \pi}} \frac{\lambda f_{0}}{D \cos (\beta)}
$$

where $\lambda$ is the wavelength of the laser source and $D$ is the lens diameter. In a well-designed system and when enough light is collected from the scene, the effect of the noise generated in the electronic circuits and the quantization noise of the peak detector on the measurement of $p$ are swamped by speckle noise.

Assuming the functions $x=g(p, \theta)$ and $z=h(p, \theta)$ have no sudden jumps in the domain around a mean value $\bar{p}$ and $\bar{\theta}$, then the means $(\bar{x}, \bar{z})$ and the variances $\left(\sigma_{x}^{2}, \sigma_{z}^{2}\right)$ can be estimated in terms of the mean, variance, and covariance of the random variables $\mathbf{p}$ and $\Theta$. The analysis of the optical arrangement together with the fact that the errors associated with the physical measurement of $p$ and $\theta$ are Gaussian random processes and are not related lead one to assume that they are uncorrelated. Therefore,

$$
\begin{aligned}
\sigma_{x}^{2} & \simeq\left(\frac{\partial g}{\partial p}\right)^{2} \sigma_{p}^{2}+\left(\frac{\partial g}{\partial \theta}\right)^{2} \sigma_{\theta}^{2} \\
\sigma_{z}^{2} & \simeq\left(\frac{\partial h}{\partial p}\right)^{2} \sigma_{p}^{2}+\left(\frac{\partial h}{\partial \theta}\right)^{2} \sigma_{\theta}^{2}
\end{aligned}
$$

where the functions $g$ and $h$ and their derivatives are evaluated at $p=\bar{p}$ and $\theta=\bar{\theta}$, and $\sigma_{p}^{2}$ and $\sigma_{\theta}^{2}$ are the variances of the spot, and the angular scanning measurement, respectively.

\subsection{Improved calibration method}

3.3.1 Table construction: As demonstrated in [4] and [5], two tables can be constructed from known positions of a calibration bar in space. A good calibration procedure should thus allow $p$ and $\theta$ to be determined accurately for all calibration plane and fiducial markings. Both methods require that one table be generated from measurements of fiducial markings on a calibration bar. These markings must be recognized by interpreting the brightness image of the bar. As of yet, no model has been proposed to quantify the effect of the light distribution on the measurement of these fiducial markings. For now, only the measurements of $p$ and $\theta$ have been sufficiently characterized. Also, because a model describing the synchronized geometry in terms of these same variables is now available (Section 2), a calibration technique that requires only the measurements of $p$ and $\theta$ along known positions of a calibration bar, without any interpretation of the brightness image, would be highly desirable. Moreover, the design equations can be used advantageously to determine the number of calibration planes and their location for look-up table construction.

One can think of an arrangement where a calibration bar is moved in such a way as to define an oblique Cartesian coordinate system as illustrated in Fig. 4. This arrangement represents a variation of the one proposed in [9]. One table is created from constant displacements of the bar in the $u$ direction and another from constant $v$ displacements. These displacements are not necessarily known exactly but sufficiently to meet the targeted calibration accuracy. Then, these two tables are transformed such that $u$ and $v$ become a function of both $p$ and $\theta$. In a practical situation, an oblique coordinate system is much easier to set up than a rectangular one. The unwarping of the tables into a rectangular coordinate system 


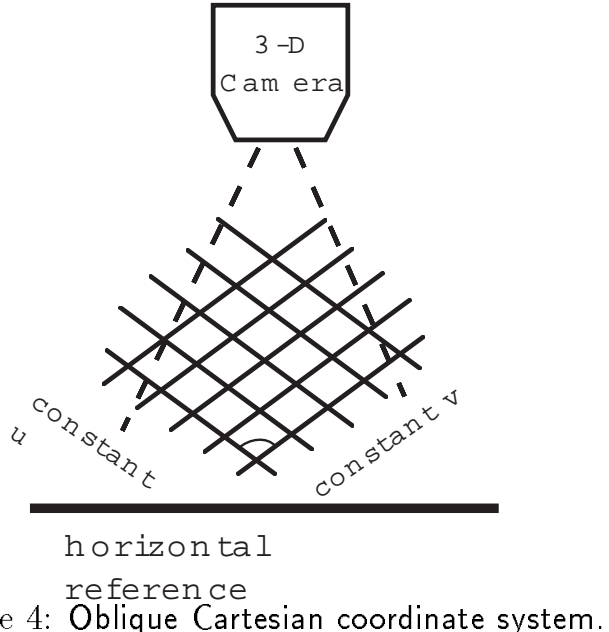

defined along some given horizon can be computed by measuring a wedge placed at different positions in the field of view. A rectangular coordinate system should yield a constant angle for the wedge. The exact spacing between horizontal and vertical lines are computed with a reference object.

3.3.2 Model-based fitting: A calibration method that tries to establish a set of tables can become very cumbersome to implement when the volume of measurement is large. A technique based on fitting a model to some calibration points in space would be of considerable interest. A model like the one developed in Section 2, i.e., (4) and (5) can be fitted to the data measured at each sampled angle in the field of view.

We propose using a linear fractional equation to calibrate a range camera for large fields of view. The chi-squared merit function is proposed in order to estimate the six parameters required for each angular position; three parameters are in the $u$ direction and three in the $v$ direction. One will minimize the following merit function for the $u$ positions

$$
\chi^{2}(\mathbf{a})=\sum_{i=1}^{N}\left[\frac{p_{i}-p\left(u_{i}, \mathbf{a}\right)}{\sigma_{i}}\right]^{2}
$$

where $\mathbf{a}$ is the parameter vector, $\sigma_{i}$ is the rms error associated with each measurement of $p_{i}$, the $u_{i}$ are known within system tolerance (e.g., accurate translation stage), and $N$ is the number of data points. The same merit function can be applied to the $v$ positions. According to theory [8], if the measurement errors on the $p$ are normally distributed, then (13) will give the maximum likelihood estimation of those parameters. Relevant intervals of confidence for those parameters can also be estimated. Once the six parameters are found for a particular angular position of the projection mirror, then simple inversion of the fractional equation can be done. The unwarping of the $(u, v)$ oblique coordinate system to a rectangular system is performed as described in Section 3.3.1.

\section{Applications}

A number of tests, some of which are reproduced here, were carried out to demonstrate the calibration techniques and were found to compare favorably to theoretical predictions. In one of these tests, the rms fluctuation of the logistic equation fitting for 512 angular positions was found to be approximately $1 / 30$ pixel. In that same test, a reference wedge was calibrated and the resulting error between true and measured edges was well within system tolerance. In another test, the estimated $P_{\infty}$ obtained from fitting a logistic equation and the theoretical model were tried for a large volume on a range camera intended for space vision applications. It features random access of any position in a field of view of $30^{\circ}$ by $30^{\circ}$ [10]. Objects located within a range of $0.5 \mathrm{~m}$ to $100 \mathrm{~m}$ can be detected. Figure 5 shows the image after calibration of a quarter-scale model (at National Research Council) of the cargo bay of the Space Shuttle Orbiter. The closest and farthest points in the field of view were at $2.6 \mathrm{~m}$ and $4.5 \mathrm{~m}$, respectively. Both estimated and measured resolution of the camera at these two locations were found to be in $x$ (along width of cargo) $125 \mu \mathrm{m}$ and $1.5 \mathrm{~mm}$ and, in $z$ (along the depth of cargo) $200 \mu \mathrm{m}$ and $4 \mathrm{~mm}$. This scale model of the cargo bay measures $4.33 \mathrm{~m}$ by $1.42 \mathrm{~m}$ by $0.6 \mathrm{~m}$.

\section{Discussion}

The critical elements of this calibration method are the validity of the model and the number and location of the calibration targets. The fractional equation of Section 2 resulted from several assumptions related to the nature of the imaging lens and the planarity and location of the mirrors. The scanning mirror is considered to be infinitely thin. Also, the galvanometer wobble and the diffraction of the laser beam were neglected. Obviously, any distortion produced by the imaging lens could be modeled. But considering the fact that the angular field of view of the imaging lens in our range cameras rarely exceeds $10^{\circ}$ for volumes of view varying between $1 \mathrm{~cm}^{3}$ and $100 \mathrm{~m}^{3}$, the pinhole assumption appears reasonable.

\section{Conclusion}

This paper has introduced a new method for the calibration of a range camera based on active triangulation. A model which is derived from the analysis of the synchronized geometry provides a basis for the method. From the analysis of the optical arrangement, some partial design guidelines are presented to assist in the initial stage of design of a range camera. Parameters like the size of the volume of view and longitudinal and range resolution can be computed. Two calibration methods are discussed. One method is based on look-up table construction. The second is aimed at range finders that can scan large volumes, i.e., $>1 \mathrm{~m}^{3}$. A logistic equation derived from the analysis is fitted with values of spot positions read from a linear position detector at a number of angular positions of the scanning mirror. An experiment involving the measurement of a quarter-scale model of the cargo bay of the Space Shuttle Orbiter was carried out. A complete raster image was obtained. 


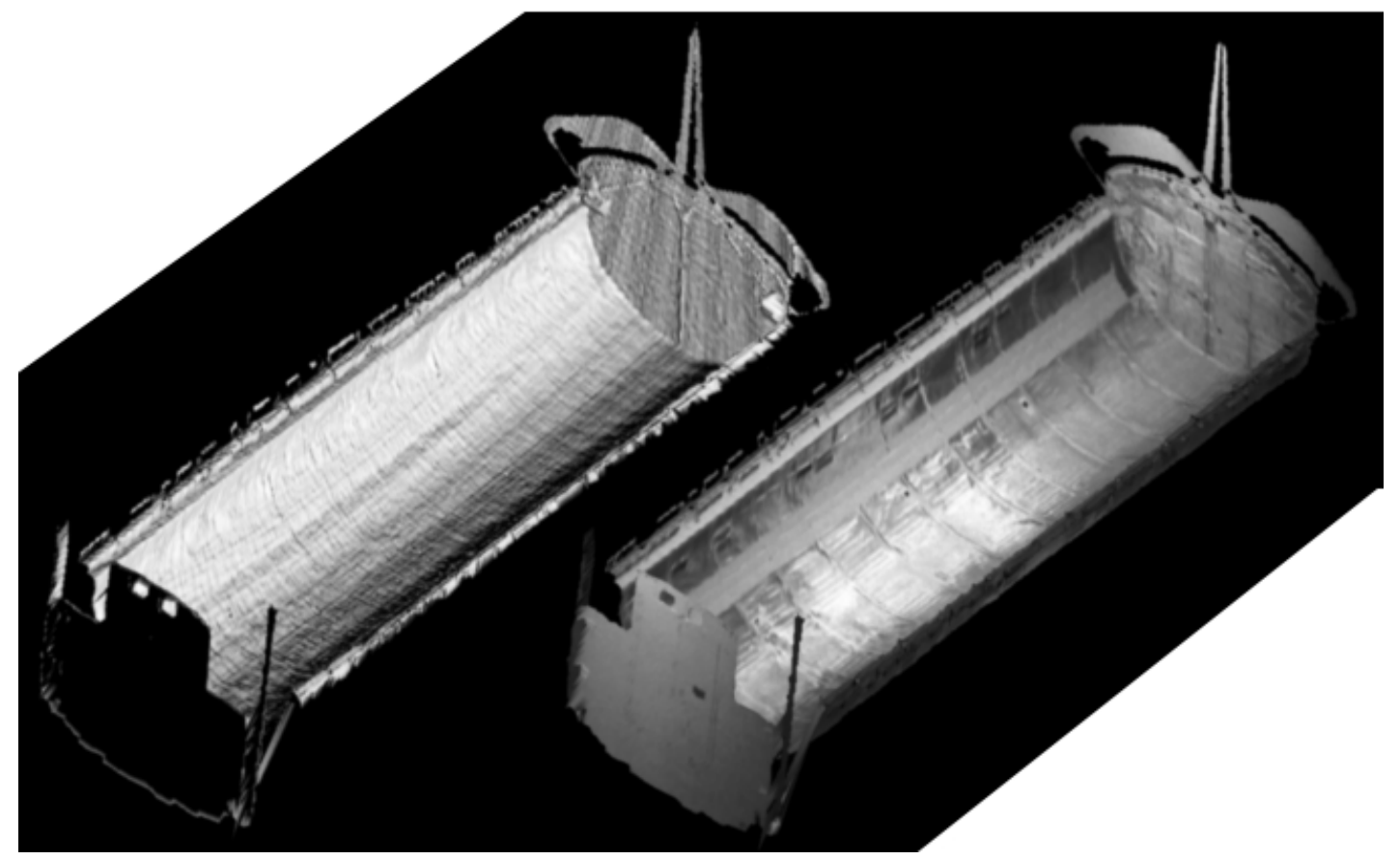

Figure 5: Two orthographic projections of a scaled cargo bay: (a) shaded, (b) mapping of intensity.

Though the analysis considered only laser range finders based upon the synchronized scanner approach other triangulation geometries with different requirements can be accommodated by a similar analysis.

\section{Acknowledgements}

The authors wish to express their gratitude to L. Cournoyer for his technical assistance provided in the course of the experiments. Finally, the authors wish to thank E. Kidd for her help in preparing the text.

\section{References}

[1] M. Rioux, "Applications of Digital 3-D Imaging," Canadian Conf. on Electr. and Comput. Eng., Ottawa, Sept. 4-6, 37.3.1-37.3.10 (1990).

[2] P.J. Besl, "Range Imaging Sensors," Machine Vision and Applic., 1, 127-152 (1988).

[3] M. Rioux, "Laser Range Finder based on Synchronized Scanners," Appl. Opt., 23, 3837-3844 (1984).

[4] F. Bumbaca, F. Blais, and M. Rioux, "Real-Time Correction of Three-dimensional Non-linearities for a Laser Range Finder," Opt. Eng. 25(4), 561565 (1986).

[5] C. Archibald and S. Amid, "Calibration of a Wrist-mounted Range Profile Scanner", Proc. of Vision Interface 89, London, Canada, June 19-23, pp. 24-28 (1989).
[6] G. Bickel, G. Hausler, and M. Maul, "Triangulation with expanded range of depth," Opt. Eng. 24(6), 975-977 (1985).

[7] F. Blais, "Control of low inertia galvanometers for high precision laser scanning systems," Opt. Eng. 27(2), 104-110 (1988).

[8] R. Baribeau and M. Rioux, "Influence of speckle on laser Range Finders," Appl. Opt., 30, 28732878 (1991).

[9] R.Schmidt, "Calibration of Three-Dimensional Space," U.S. Patent \#4682894 (Jul. 1987).

[10] F.Blais, M.Rioux, and S.G.Mclean, "Intelligent, Variable Resolution Laser Scanner for the Space Vision System," SPIE 1482, 473-479 (1991). 